\title{
Time Lapse Spectropolarimetry: Constraining the Nature and Progenitors of Interacting CCSNe
}

\author{
Leah Huk ${ }^{1}$ \\ ${ }^{1}$ Dept. of Physics \& Astronomy, University of Denver, \\ 2112 E. Wesley Ave., Denver, CO 80210, USA \\ email: leah.huk@gmail.com
}

\begin{abstract}
SNe of Type IIn are among the brightest supernova explosions due to strong circumstellar interaction. Examining the geometric and optical properties of the circumstellar material (CSM) can help to identify the progenitors of individual IIn SNe. Polarimetry is the optimal method for constraining CSM characteristics, as polarimetric signals both depend upon and preserve geometric information from unresolved sources. I present the results of fitting an ensemble of simulated polarized $\mathrm{H} \alpha$ emission-line profiles of interacting $\mathrm{SNe}$, created using a three-dimensional Monte Carlo radiative transfer code called SLIP, to the multi-epoch observed polarized spectra of the Type IIn SN 1997eg. Further study of this model ensemble will allow us to investigate relationships among SNe IIn based on viewing angle and consider how the category should be subdivided based on physical properties of the CSM and/or progenitor.
\end{abstract}

Inclination is the most influential parameter in our fits. The models best fitting the observed continuum polarization magnitude and depolarization at $\mathrm{H} \alpha$ line center are constrained to inclinations near $90^{\circ}$, implying a nearly edge-on orientation for SN $1997 \mathrm{eg}$.

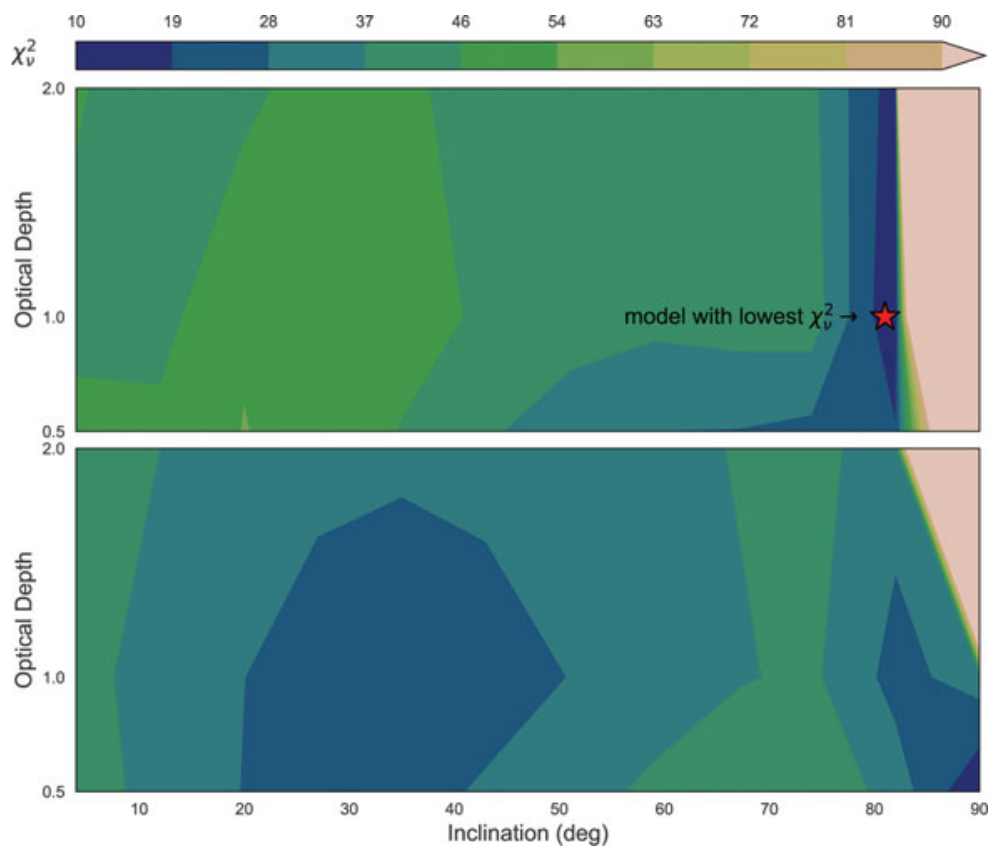

Figure 1. Reduced $\chi^{2}$ values for fits to the polarized H $\alpha$ line of SN 1997eg at day 16, with light arising from a central source (top) and from a shock region interior to the CSM (bottom). 